\title{
STELLAR PULSATION: (I) ANALYSIS OF \\ STABILITY OF ENVELOPE
}

\author{
YU ZHI-YAO \\ Shanghai Observatory, 80 Nandan Road, Shanghai 200030, China
}

\begin{abstract}
The life-time of the star on AGB is approximately $6 \times 10^{4} \mathrm{yr}$. We divide it into front half and back half of AGB (including to optical Mira variable and $\mathrm{OH} / \mathrm{IR}$ star) according to their evolution character. The observations show that the star has nonpulsation, but constant mass loss rate $\left(\sim 5 \times 10^{-7} M_{\odot} \mathrm{yr}^{-1}\right)$ on front half of AGB. Its circumstellar envelope is formed. When the star has pulsation on back half of AGB, its mass loss rate is relative with time, and increases gradually. In this time the star is on the stage of optical Mira variable. When the mass loss rate reaches the value of $\sim 3 \times 10^{-6}$ $M_{\odot} \mathrm{yr}^{-1}$, the star evoluted from the stage of optical variable into the stage of radio bright

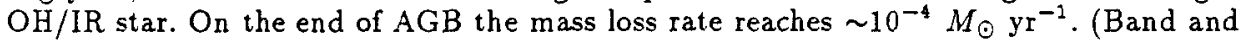
Habing 1983, Hermen and Habing 1985).
\end{abstract}

The contribution of the star to its circumstellar envelope are its radiation pressure $F_{R}$ and gravitation. Here we assume its radiation pressure is first, and the gravitation is secondary. So we neglect the disturb of gravitation. We give the characters of the disturb as following.

1. FRONT HALF STAGE ON AGB

The disturb of $F_{R}$ are not relative with time.

2. BACK HALF STAGE ON AGB

(1) The Stage of Optical Mira Variable

The disturb of $F_{R}$ are periodicity.

(2) The Stage of OH/IR Star

The disturb of $F_{R}$ raises with time gradually.

Assuming the circumstellar envelope is ideal gas and it is radial spheresymmetrical adiabatic out-flowing, we have obtained and studied the equations of the disturbance of the stable state for the envelope, and the following conclusions have been obtained.

(1) The star has non-pulsation on front half of AGB, but constant mass loss rate $\left(\sim 5 \times 10^{-7} M_{\odot} \mathrm{yr}^{-1}\right)$. The solutions of stable state are Lyapunov stability.

(2) When the star is on the stage of optical Mira variable it has the pulsation, its mass loss rate is approximately between $5 \times 10^{-7}$ and $3 \times 10^{-6}$ $M_{\odot} \mathrm{yr}^{-1}$, and the all disturbance solutions of velocity, density, and pressure of gas in the envelope, pure heat got by its gas per unit volume, and its mass loss rate have the character of spheric-wave. If we fix on time $t$, the all disturbance solutions are functions, which values are attenuate with increase of radius $r$. If we fix on $r$, the all disturbance solutions are the harmonic functions of $\omega_{*} t\left(\omega_{*}\right.$ is $2 \pi / T_{*}, T_{*}$ is the optical period of the star). 
(3) When the star is on the stage of OH/IR star it has the pulsation, its mass loss rate is approximately from $3 \times 10^{-6} M_{\odot} \mathrm{yr}^{-1}$ to $1 \times 10^{-4} M_{\odot} \mathrm{yr}^{-1}$, the all disturbance solutions increase with increase of time, and solutions of stable state are non-stability. Our computational results indicate that the mass loss rate raises gradually. On the end of AGB the mass loss rate is $1.2 \times 10^{-4} M_{\odot} \mathrm{yr}^{-1}$. The circumstellar envelope is non-stability.

\section{References}

Band, B. and Habing, H. J.: 1983, Astronomy and Astrophysics 127, 73.

Hermen, J. and Habing, H. J.: 1985, Phys. Rep. 124, 175. 